\title{
Non-invasive measurement of liver iron concentration by magnetic resonance imaging and its clinical usefulness
}

Jose M. Alustiza' ${ }^{1}$ Agustin Castiella², Eva Zapata ${ }^{2}$, Iratxe Urreta ${ }^{3}$, Emma Salvador ${ }^{1}$, Jose I. Emparanza ${ }^{3}$

${ }^{1}$ Radiology Service, Osatek Donostia, Donostia, Spain

${ }^{2}$ Gastroenterology Service, Donostia University Hospital, Donostia, Spain

${ }^{3}$ Clinical Epidemiology Unit, CASPe, CIBER-ESP, Donostia University Hospital, Donostia, Spain

Submitted: 4 January 2020

Accepted: 16 March 2020

Arch Med Sci

DOI: https://doi.org/10.5114/aoms/119118

Copyright (c) 2021 Termedia \& Banach
Corresponding author: Agustin Castiella PhD, MD Gastroenterology Service Donostia University Hospital $\mathrm{P}^{\mathrm{a}}$ Dr. Beguiristain $\mathrm{s} / \mathrm{n}$ 20014 Donostia, Spain Phone: 00-34-943007000 Fax: 00-34-943-032856 E-mail:

agustincastiella@yahoo.es

\begin{abstract}
A bstract
Determination of liver iron concentration by magnetic resonance imaging (MRI) is becoming the new technique of choice for the diagnosis of iron overload in hereditary haemochromatosis and other liver iron surcharge diseases. Determination of hepatic iron concentration obtained by liver biopsy has been the gold standard for years. The development of MRI techniques, via signal intensity ratio methods or relaxometry, has provided a non-invasive and more accurate approach to the diagnosis of liver iron overload.

This article reviews the available MRI methods for the determination of liver iron concentration and also evaluates the technique for the diagnosis and quantification of iron overload in different clinical practice scenarios.
\end{abstract}

Key words: liver, ferritin, liver iron concentration, determination, magnetic resonance imaging $(\mathrm{MRI})$.

\section{Introduction}

Liver iron overload is defined as a liver iron concentration (LIC) greater than $36 \mu \mathrm{mol} \mathrm{Fe} / \mathrm{g}$, which can cause liver disease, and the aetiologies include inherited causes of iron overload, secondary iron overload and miscellaneous causes such as alcohol, inflammation, cytolysis and metabolic syndrome. Hereditary haemochromatosis $(\mathrm{HH})$ is a common autosomal recessive disease characterized by mutations in the HFE gene. Approximately $85-90 \%$ of patients who have inherited forms of iron overload are homozygous for the C282Y mutation in the HFE gene, with a small minority who are compound heterozygotes [1]. Other mutations in genes regulating iron metabolism (TfR2, HJV, SLC4OA1) produce rarer cases of inherited haemochromatosis [2].

Hereditary haemochromatosis is characterized by an increase in gastrointestinal absorption of iron, which can lead to deposits of iron in the liver, pancreas, heart, skin and joints. Phenotypic expression of $\mathrm{HH}$ causes progressive iron overload in the liver, the target organ of the disease, with a high risk of liver fibrosis, cirrhosis and hepatocellular carcinoma [1]. When iron overload is due to increased destruction of red blood cells, the disease is called secondary haemochromatosis or 
haemosiderosis. Some hepatopathies, by an unknown mechanism, can also generate iron overload [2].

Hereditary haemochromatosis is increasingly being recognized by clinicians but is still underdiagnosed because most people have no symptoms in the early stage of the disease [1]. It is therefore important to diagnose the disease before there is any organ involvement due to iron accumulation in various tissues [1].

Clinical penetrance in $\mathrm{HH}$ (biochemical penetrance with symptoms and/or organ damage) occurs more frequently in men than in women: $28.4 \%$ of men and $2.4 \%$ of women with $\mathrm{C} 282 \mathrm{Y}$ homozygosity had liver iron overload disease [1].

In $\mathrm{HH}$, treatment with phlebotomy is highly effective [2]. Patients with haemosiderosis often have anaemia and are treated with iron chelators [2]. The first step toward diagnosis is measuring the transferrin saturation index (TSI) and the blood ferritin levels. Both are high in the presence of iron overload [2]. When $\mathrm{HH}$ is suspected, a genetic study of $\mathrm{C} 282 \mathrm{Y}$ and H63D mutations of the HFE gene is conducted to rule out the most common form of $\mathrm{HH}[3,4]$. The standard method for direct assessment of total body iron is to measure the $\operatorname{LIC}[1,2,5]$. The normal LIC value is $<36 \mu \mathrm{mol} \mathrm{Fe} / \mathrm{g}$. In $\mathrm{HH}$ and haemosiderosis it is usually $>80 \mu \mathrm{mol} \mathrm{Fe} / \mathrm{g}$, except in liver iron overload secondary to hepatopathies $[2,4,6]$; therefore measurement of LIC is the gold standard for the diagnosis of haemochromatosis. For years, LIC was estimated by liver biopsy using spectrophotometry. Liver biopsy provides direct assessment of LIC and can also be used for fibrosis staging and ruling out concurrent liver diseases, but there is a slight risk of complications, such as bleeding or sample limitation and variability, leading to misinterpretations.

Ultrasound imaging does not allow detection or quantification of liver iron overload. Computed tomography allows detection of iron overload by demonstrating increased attenuation of the liver parenchyma but is not sensitive or specific enough for grading or quantification of iron overload. However, magnetic resonance imaging (MRI) determination of LIC has demonstrated an excellent correlation with liver biopsies $[6,7]$.

Nowadays, MRI is the best non-invasive technique to determine LIC [5]. This article reviews the available MRI techniques for determining LIC accurately today and evaluates this non-invasive technique for the diagnosis and quantification of iron overload in different clinical practice scenarios. Haemochromatosis secondary to haematological disorders has not been included.

\section{Evaluation of liver iron concentration by magnetic resonance imaging}

The role of MRI in the detection of liver iron overload is based on the paramagnetic effect of iron in the liver, shortening the transversal relaxation rate and thereby reducing the signal intensity of the hepatic parenchyma in MR images.

To obtain an MR image implies a succession of excitations and relaxations of the hydrogen nuclei in the patient's body. After one excitation, the transversal relaxation rate is defined by the time it takes for the transverse magnetization to reach $37 \%$ of its original magnitude. When the curve is evaluated by means of spin echo sequences it is called 'T2' but if gradient echo sequences are used it is called ' $\mathrm{T} 2$ "'. Both $\mathrm{T} 2$ and $\mathrm{T} 2$ " values are measured in milliseconds.

Iron overload accelerates the transversal relaxation curve, shortening $\mathrm{T} 2$ and $\mathrm{T} 2^{*}$ values in proportion to the quantity of iron and decreasing the signal intensity of the liver in the MR images. Some studies have strongly demonstrated this correlation between the measured liver signal, the T2 or T2* values and LIC $[6-12,13]$. The T2 and $\mathrm{T} 2^{*}$ values are often converted to their reciprocals, R2 (1000/T2) and R2* $\left(1000 / 2^{*}\right)$, measured in hertz per second.

All the studies that have compared MRI with liver biopsy for LIC determination have found a very high correlation, with $86-94 \%$ sensitivity and $81-100 \%$ specificity, for the diagnosis of moderate and high overload $[6,7,11]$.

There are thus two different methods for quantifying LIC by MRI: relaxometry methods to evaluate T2 or T2*; and SIR methods to evaluate the signal of the liver parenchyma in MRI.

\section{Relaxometry methods}

Relaxometry methods calculate $\mathrm{T} 2 / \mathrm{T} 2^{*}$ transversal relaxation rates. To accurately plot the transversal relaxation curve it is necessary to have the maximum number of points on the curve over the relaxation time. Each measured point corresponds to one echo. Nowadays, multi-echo sequences are performed with a large number of increasing echoes for a few seconds. The first echo time is called TE, 'echo train' is the total number of echoes and 'interval TE' is the time between two echoes. The first TE must be very short in this method. High iron overload implies very fast changes in the relaxation curve and a very short TE is necessary in order to evaluate these early changes with the acceleration of the curve (Figure 1). The TE values are about $1 \mathrm{~ms}$ for the $\mathrm{T} 2^{*}$ methods and-approximately $4 \mathrm{~ms}$ for the $\mathrm{T} 2$ methods.

Recently, several T2/T2* relaxometry methods have been validated to quantify LIC accurately, 


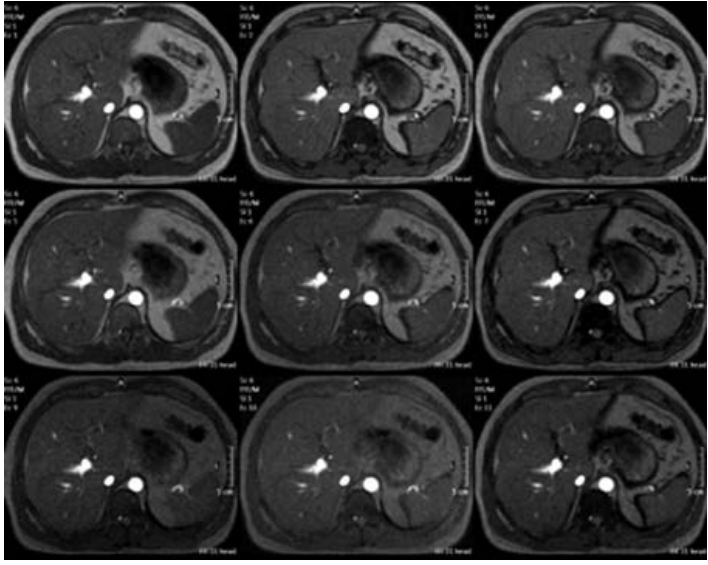

A

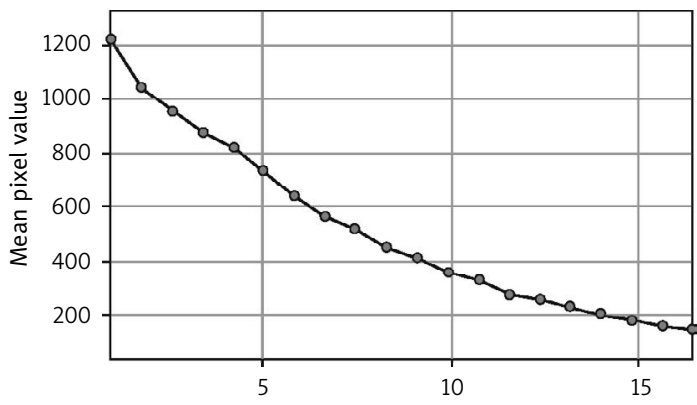

Echoes

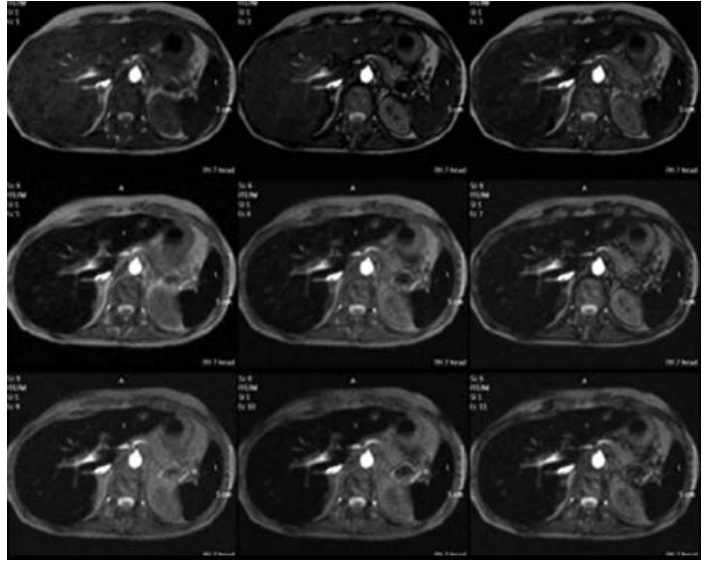

B

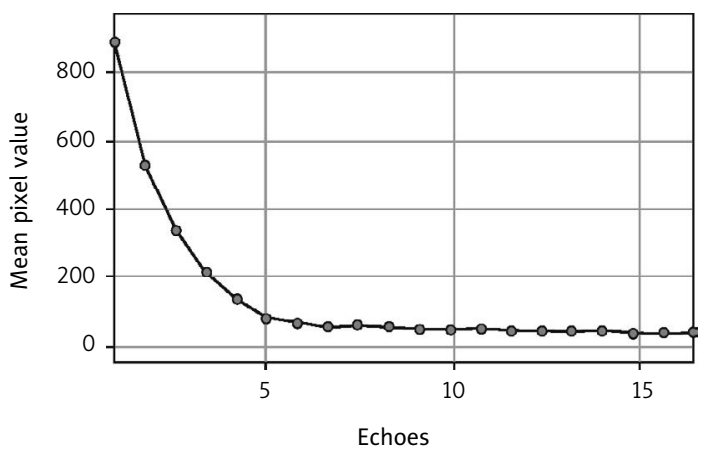

Figure 1. T2* relaxometry. Gradient multi-echo sequence, with 19 echoes (TR $21 \mathrm{~ms}$, flip angle $35^{\circ}$, first echo 0.9 ms, TE interval $1 \mathrm{~ms}$ ). Magnetic resonance image of the first nine echoes and graph of the T2* curve. A - Patient without iron overload: liver isosignal in all the echoes and T2 = $11.5 \mathrm{~ms}$ (normal T2 curve). B - Polytransfused patient with acute myeloblastic leukaemia: high iron overload, $\mathrm{T}^{*}=2.5 \mathrm{~ms}$; reduced liver signal visible from the first echoes; T2* curve different to patient A with a vertical first component of the curve

using quantification of LIC on liver biopsies by spectrophotometry as a gold standard. Moreover, they have calculated mathematical formulas to transform T2/T2* values into LIC units, expressed in $\mu \mathrm{mol} \mathrm{Fe/g} \mathrm{[8-11,} \mathrm{13].}$

Relaxometry methods are able to discriminate all the ranges of iron overload; furthermore, $\mathrm{T}^{*}$ methods are able to measure the iron concentration in the myocardium.

The most used $T 2$ relaxometry method is Ferriscan (www.resonancehealth.com), approved by the U.S. Food and Drug Administration (FDA). An advantage of this method is that the correct quality of acquisition technique and post-processing is guaranteed because all calculations are done remotely from where the method was designed, in Australia. It has been the reference method in the majority of international multicentre clinical trials evaluating new iron chelant drugs in patients with haemosiderosis. However, the drawback is that the acquisition sequences are long and cannot be performed in apnoea, increasing the acquisition time and worsening the quality of images due to movements of the liver during breathing; it has an added cost per patient and two days are needed to obtain results.
The $T 2^{*}$ relaxometry methods are acquired in breath-hold with shorter gradient echo sequences, giving better images without respiration artefacts. R2* values have a linear correlation with LIC $[8,10]$ and Garbowski (www.CMRtools.com) and Wood's methods are the most widely used. Hankins and Henninger validated other $\mathrm{T}^{*}$ relaxometry methods $[9,13]$ with mathematical models to calculate LIC with R2* values.

Relaxometry methods have some differences in the acquisition parameters and in post-processing but all the mathematical formulas for calculating LIC in $\mu \mathrm{mol} \mathrm{Fe} / \mathrm{g}$ are very similar. All are reproducible in different machines when the acquisition and post-processing parameters of the method are exactly reproduced $[11,14-16]$. There is still no agreement on a single universal method. However, a new acquisition sequence and post-processing method that takes into account the most important validated methods (www.MRquantif.org) has been designed recently by the University of Rennes [17, 18] and it is an interesting platform to compare different methods and move towards a universal language of $\mathrm{T}^{*}$ relaxometry techniques.

Iron overload in the liver can be associated with steatosis in very different kinds of diseas- 
Ref. Mr Center

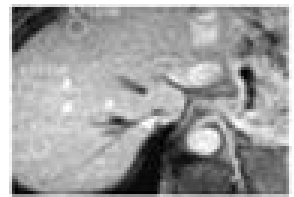

B

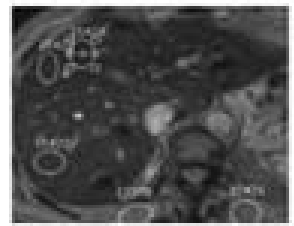

$\mathrm{C}$

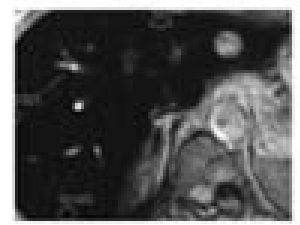

MR Unit 1

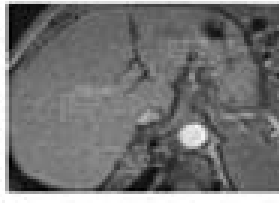

MR Unit 2

MR Unit 3

MR Unit 4
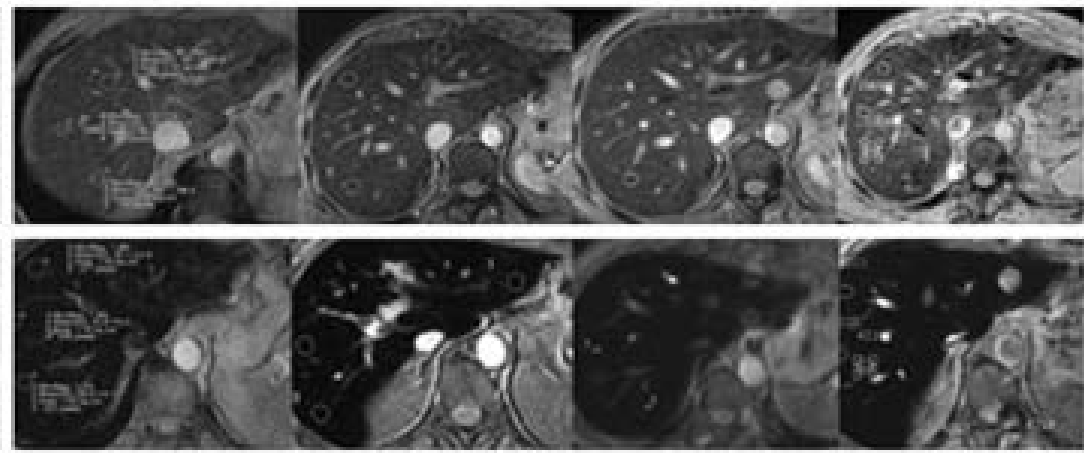

Figure 2. Reproducibility of quantification of liver iron concentration (LIC) by magnetic resonance imaging (MRI) with the signal intensity ratio (SIR) method. Three patients with different levels of LIC, each studied in five different MRI machines of 1.5 Tesla using the same method. Magnetic resonance image: gradient echo proton density sequence, TR $120 \mathrm{~ms}$, TE $4 \mathrm{~ms}$, flip angle $20^{\circ}$. Reference MR centre corresponds to the centre in which the method was validated [6]. Magnetic resonance units 1-4 correspond to four other machines located in the same city. A - Patient without iron overload: normal signal of the liver; LIC measured in each centre was 22, 15, 7, 14 and $10 \mu \mathrm{mol} \mathrm{Fe} / \mathrm{g}$, respectively. B - Patient with moderate overload: slight decrease in liver signal; measured LIC values were $65,66,60,56$ and $61 \mu \mathrm{mol} \mathrm{Fe/g.} \mathrm{C} \mathrm{-} \mathrm{Patient} \mathrm{with} \mathrm{high} \mathrm{iron} \mathrm{overload:} \mathrm{large} \mathrm{decrease} \mathrm{in} \mathrm{liver} \mathrm{signal;} \mathrm{measured}$ LIC values were 249, 249, 270, 251 and $287 \mu \mathrm{mol} \mathrm{Fe/g}$

es: metabolic syndrome and chronic hepatopathy [19], thalassaemia [20] and haemodialysis [21]. The presence of fat can alter the MRI estimation of $\mathrm{LIC}$, especially in $\mathrm{T}^{*}$ relaxometry methods. To minimize this bias, new multi-echo sequences are able to evaluate the fat fraction and give a 'corrected' $T 2^{*}$ value simultaneously. This type of sequence is known as a proton density fat fraction (PDFF). MRI vendors are optionally proposing their own versions of this technique: General Electric, 'IDEAL-IQ'; Philips, 'mDixon-Quant'; Siemens, 'LiverLab'; Toshiba, 'MR Body Expert'. These methods are already validated to estimate the fat fraction and some have already been evaluated for determining LIC, with promising results [22]. Although there are some differences between vendors, in the near future when they are widely accessible these sequences will be the solution to the interference between iron and fat.

Several studies that have correlated MRI with liver biopsies have found that the presence of fibrosis in the liver does not have a significant impact on the precision of LIC estimation by MRI [7, 23].

\section{Signal intensity ratio methods}

In SIR methods the signal intensities of the liver and paraspinal muscle are measured directly on the MR image. The muscle is taken as a reference tissue because it does not accumulate iron and the ratio of the liver signal intensity to that of the paraspinal muscle is calculated. Gradient echo sequences are used because of their greater sensitivity to the paramagnetic effect of iron. Thus, the difference between the liver and muscle signal intensity values basically depends on the value of iron overload. In order to be able to quantify different levels of iron overload more than one sequence is necessary $[6,7]$. A very important technical aspect for all SIR methods is that the acquisition of quantification sequences must be performed with a body coil. A surface coil can be placed on the patient and even connected for other sequences of the study but it should not be activated for the acquisition of quantification sequences [17]. There is a major risk of erroneous calculation of LIC with these SIR methods.

Estimation of LIC by an SIR method is easier to perform than $\mathrm{T} 2$ relaxometry methods $[6,7]$. The most significant advantage of SIR methods is that they are very accessible, all methods being feasible in every 1.5-tesla machine in the world. Moreover, acquisition technique and post-processing are standardized and the results are reproducible in different machines [24] (Figure 2). The limitations of SIR methods compared to T2 relaxometry methods are that they become saturated at very high iron overload and they cannot evaluate iron overload in the myocardium.

Various SIR methods have been validated by liver biopsies and they have produced a formula to calculate LIC $[6,7,12]$. The method from the University of Rennes (France), published in 2004, has been used worldwide [7]. Several studies have 
shown that it overestimates patients with moderate overload [25-27] and recently it has been replaced by a new $\mathrm{T}^{*}$ relaxometry method (WWW. MRQuantif.org) that is also able to perform SIR calculations. Additionally, the Spanish Society of Abdominal Imaging (SEDIA, www.sedia.es) has developed a specific mathematical model to estimate LIC that shows a good correlation with relaxometry techniques [26, 27] (https://imagemed. univ-rennes1.fr/en/mrquantif/quantif.php). Both of these SIR methods [6, 7] saturate with very high iron overload, which means that they correctly recognize patients with high iron overload and that they should be treated but they are not precise in this range.

To overcome this saturation with high iron overload, a third method was developed by the University of Lille, France [12], specifically for patients with very high iron overload (http://oernst.f51vg. free.fr/liver/iron.html).

\section{Evaluation of liver iron concentration using magnetic resonance imaging in clinical practice}

Despite the scientific evidence generated during the last three decades showing the accuracy of MRI for evaluating LIC, there is still no agreement about which patients should be scanned by MRI in order to rule out iron overload.

\section{Patients with hyperferritinaemia}

The vast majority of patients with hyperferritinaemia do not present iron overload in the liver $[28,29]$. However, it is important to recognize which of them have iron overload in order to be treated by phlebotomy [19, 30-33]. This treatment does not offer any benefit to patients without iron overload and, moreover, it can generate some complications [19, 34].

Four causes account for more than $90 \%$ of cases of hyperferritinaemia: alcohol, inflammation, cytolysis (hepatitis, neoplasms) and metabolic syndrome [28]. In general the clinical context (such as being overweight or hypertensive), some analytical parameters (hypertransaminasaemia, C-reactive protein, hyperlipidaemia, hyperglycaemia) [28, 29] and the absence of elevation of the TSI are enough for diagnosis and for ruling out iron overload. A TSI of $>45 \%$ has a sensitivity of $89 \%$ (specificity $61 \%$ ) for iron overload in these patients $[4,35]$. In patients with metabolic syndrome, iron overload is suspected when ferritin is $>450 \mu \mathrm{g} / \mathrm{l}[29,36]$.

However, in clinical practice diagnosis is often difficult. In $40-50 \%$ of cases different pathologies able to generate hyperferritinaemia are simultaneously associated $[28,29]$. Dysmetabolic iron over- load syndrome is a disorder that can be present in patients with metabolic syndrome characterized by moderate iron overload $(<150 \mu \mathrm{mol} \mathrm{Fe} / \mathrm{g})$, often with a normal TSI [36]. Recently, haemodialysis-associated haemosiderosis has been encountered in nearly $50 \%$ of dialysis patients and serum iron markers are inaccurate for guiding iron repletion in chronic kidney disease [37].

Thus, there is no combination of ferritin and TSI values that can confirm or rule out iron overload in patients with hyperferritinaemia. Therefore, in cases where it is difficult to determine the cause, when several possible aetiologies are present simultaneously or when the level of ferritin tends to increase over time, hepatic MRI is indicated to evaluate the presence of iron overload $[28,38]$.

\section{Iron overload secondary to liver disease}

In the case of cutaneous porphyria, the benefit of the depletion of iron overload through phlebotomy is demonstrated [3]. However, with alcoholic liver disease no benefit has been shown [3]. Also, with hepatitis C, if the LIC is < $45 \mu \mathrm{mol} \mathrm{Fe/g} \mathrm{[3]}$ then the patient should not be treated with phlebotomy. Several studies have shown that phlebotomy is not indicated in patients with liver disease if they do not have iron overload [19, 33, 34].

In these patients with liver disease, therefore, iron overload should always be confirmed using MRI before initiating a treatment with phlebotomy $[34,38]$.

\section{Patients with hereditary haemochromatosis}

According to some clinical guidelines [2-4] the diagnosis of $\mathrm{HH}$ linked to the HFE gene is made when simultaneous elevation of ferritin and TSI and C282Y HFE mutation in homozygosis are observed. However, not all patients with this mutation and simultaneous elevation of ferritin and TSI have a defined disease and they must only be treated if liver iron overload is demonstrated.

The cut-off point to define high iron overload in the range of haemochromatosis is $80 \mu \mathrm{mol} \mathrm{Fe/g}$ or a liver iron index of $>1.9[2,4]$. The cut-off point for significant iron overload is $60 \mu \mathrm{mol} \mathrm{Fe/g}$. It has been shown that with this level of LIC there is activation of stellate cells, which causes liver fibrosis [39]. Therefore, in these patients treatment with phlebotomy is required when the LIC is $>60 \mu \mathrm{mol}$ $\mathrm{Fe} / \mathrm{g}[40]$.

Most of the guidelines recognize that it is necessary to verify the presence of actual iron overload before starting treatment with phlebotomy and that it is contraindicated in the absence of iron overload $[4,31,33,41]$. There is also agreement that $M R I$ recognizes iron overload accurately $[2,4,42]$ and that liver biopsy is no longer 


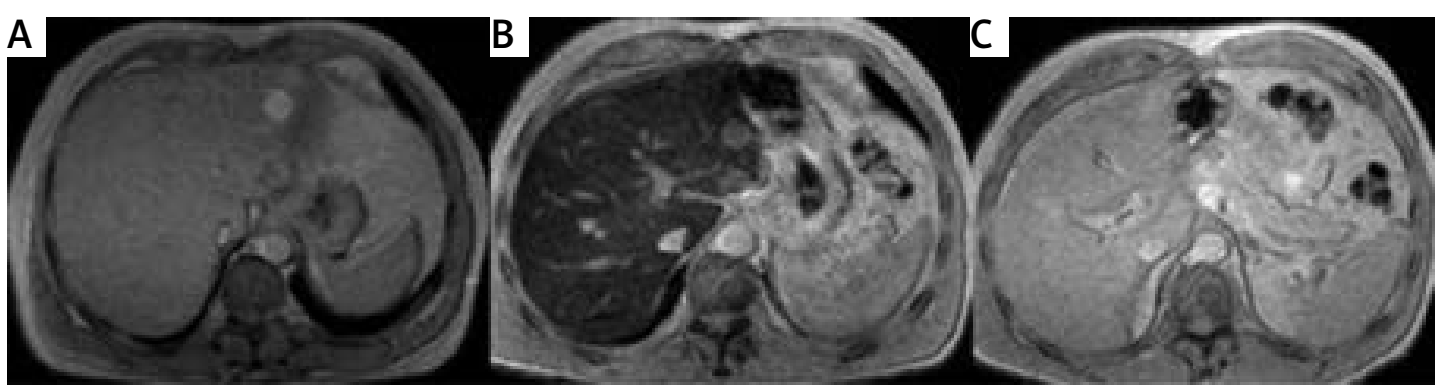

Figure 3. Two patients with C282Y/H63D mutation. A - Male, 52 years old, asymptomatic, ferritin $850 \mu \mathrm{g} / \mathrm{l}$, transferrin saturation index (TSI) $55 \%$, consumption of alcohol. Abdominal lymphoma discovered when performing hepatic magnetic resonance imaging (MRI) to evaluate liver iron concentration. Magnetic resonance image: gradient echo proton density (TR $120 \mathrm{~ms}$, TE $4 \mathrm{~ms}$, flip angle $20^{\circ}$ ). Normal signal of the liver, without iron overload. Liver iron concentration measured by MRI using signal intensity ratio (SIR) method: $12 \mu \mathrm{mol} \mathrm{Fe} / \mathrm{g}$. B - Asymptomatic 55 -year-old male, ferritin $650 \mu \mathrm{g} / \mathrm{l}$, TSI 60\%, no risk factors for liver disease. Magnetic resonance image: gradient echo proton density (TR $120 \mathrm{~ms}$, TE $4 \mathrm{~ms}$, flip angle $20^{\circ}$ ). Decreased liver signal in relation to high iron overload. LIC measured by MRI using SIR method: $120 \mu \mathrm{mol} \mathrm{Fe/g.} \mathrm{C} \mathrm{-} \mathrm{Patient} \mathrm{B} 1$ year later, after treatment with phlebotomy and normalization of ferritin and TSI. Magnetic resonance image: gradient echo proton density (TR $120 \mathrm{~ms}$, TE $4 \mathrm{ms,}$ flip angle $20^{\circ}$ ). Normal signal of the liver, with no iron overload. Liver iron concentration measured by MRI using SIR method: $19 \mu \mathrm{mol} \mathrm{Fe} / \mathrm{g}$

indicated for this purpose. Therefore, we can say that MRI with quantification of LIC is indicated in all patients diagnosed with haemochromatosis linked to the HFE gene before starting treatment. The level of LIC can predict the extent of the phlebotomy [2] and also the risk of liver fibrosis [43]. Magnetic resonance imaging may be used for follow-up after treatment.

In patients with elevated ferritin and TSI along with $\mathrm{C} 282 \mathrm{H} / \mathrm{H} 63 \mathrm{D}$ or $\mathrm{H} 63 \mathrm{D} / \mathrm{H} 63 \mathrm{D}$ mutations, it is considered that without other added factors these predisposing mutations do not generate high iron overload [1, 2]. Therefore, MRI will be indicated to decide whether or not the patient should be treated with phlebotomy (Figure 3).

In patients with a negative genetic study, more advanced genetic studies should not be performed without iron overload having been previously demonstrated by $\mathrm{MRI}[2,4,41]$, and evaluation of LIC in these cases is indicated after eliminating other possible causes of elevated ferritin and TSI, such as liver disease, alcoholism, steatohepatitis or inflammation $[1,2]$.

\section{Liver iron concentration determination and fibrosis prediction in hereditary haemochromatosis patients}

The risk of significant fibrosis or cirrhosis has been associated with the level of LIC $[38,43]$. Bassett et al. [44] introduced the concept of a threshold for LIC above which cirrhosis was more likely, and Sallie et al. [45] reported that, in addition to LIC, age over 45 years may be a risk factor for significant fibrosis or cirrhosis. Olynyk et al. [43] demonstrated that the duration of hepatic iron exposure, manifested by time and LIC, may be very relevant to the development of significant liver fibrosis. If the product of age and LIC (the fibrosis index) obtained by liver biopsy or by MRI is higher than 480,000 , this results in $100 \%$ sensitivity and $86 \%$ specificity for the diagnosis of high-degree fibrosis (F3-F4) [43]. This fibrosis index has been validated externally, with good results [46].

\section{Conclusions}

The management of patients with suspected liver iron overload remains complicated [38]. Magnetic resonance imaging is an accurate, validated, reproducible and accessible diagnostic tool used to assess the actual presence of iron overload in the liver. The MRI technique should be used specifically in each of the different clinical contexts to reduce uncertainty in the management of these patients and treatment with phlebotomy should be used only for those with actual iron overload.

\section{Conflict of interest}

The authors declare no conflict of interest.

\section{References}

1. Milman NT, Schioedt FV, Junker AE, Magnussen K. Diagnosis and treatment of genetic HFE-hemochromatosis: the Danish aspect. Gastroenterol Res 2019; 12: 221-32.

2. Fitzsimons EJ, Cullis JO, Thomas DW, Tsochatzis E, Griffiths WJH; on behalf of the British Society for Hematology. Diagnosis and therapy of genetic haemochromatosis (review and 2017 update). Br J Haematol 2018; 181: 293-303.

3. Bacon BR, Adams PC, Kowdley KV, Powell LW, Tavill AS. Diagnosis and management of hemochromatosis: 2011 practice guideline by the American Association for the Study of Liver Diseases. Hepatology 2011; 54: 328-43.

4. European Association for the Study of the Liver. EASL clinical practice guidelines for HFE hemochromatosis. J Hepatol 2010; 53: 3-22. 
5. Chin J, Powell LW, Ramm LE, Ayonrinde OT, Ramm GA, Olynyk JK. Utility of hepatic or total iron burden in the assessment of advanced hepatic fibrosis in HFE hemochromatosis. Sci Rep 2019; 9: 20234.

6. Alustiza JM, Artetxe J, Castiella A, et al.; Gipuzkoa Hepatic Iron Concentration by MRI Study Group. MR quantification of hepatic iron concentration. Radiology 2004; 230: 479-84

7. Gandon Y, Olivie D, Guyader D, et al. Non-invasive assessment of hepatic iron stores by MRI. Lancet 2004; 363: 357-62.

8. Garbowski MW, Carpenter JP, Smith G, et al. Biopsybased calibration of $\mathrm{T}^{*}$ magnetic resonance for estimation of liver iron concentration and comparison with R2 Ferriscan. J Cardiovasc Magn Reson 2014; 16: 40.

9. Hankins JS, McCarville MB, Loeffler RB, et al. R2* magnetic resonance imaging of the liver in patients with iron overload. Blood 2009; 113: 4853-5.

10. Wood JC, Enriquez C, Ghugre N, et al. MRI R2 and R2* mapping accurately estimates hepatic iron concentration in transfusion-dependent thalassemia and sickle cell disease patients. Blood 2005; 106: 1460-5.

11. St Pierre TG, Clark PR, Chua-anusorn W, et al. Noninvasive measurement and imaging of liver iron concentrations using proton magnetic resonance. Blood 2005; 105: 855-61.

12. Rose C, Vandevenne P, Bourgeois E, Cambier N, Ernst O. Liver iron content assessment by routine and simple magnetic resonance imaging procedure in highly transfused patients. Eur J Haematol 2006; 77: 145-9.

13. Henninger $\mathrm{B}$, Zoller $\mathrm{H}$, Rauch $\mathrm{S}$, et al. R2* relaxometry for the quantification of hepatic iron overload: biopsy-based calibration and comparison with the literature. Rofo 2015; 187: 472-9.

14. Kirk P, He T, Anderson LJ, et al. International reproducibility of single breathhold T2* MR for cardiac and liver iron assessment among five thalassemia centers. J Magn Reson Imaging 2010; 32: 315-9.

15. Westwood MA, Anderson LJ, Firmin DN, et al. Interscanner reproducibility of cardiovascular magnetic resonance $\mathrm{T}^{\star}$ measurements of tissue iron in thalassemia. J Magn Reson Imaging 2003; 18: 616-20.

16. Meloni A, Ramazzotti A, Positano V, et al. Evaluation of a web-based network for reproducible $T 2^{*} \mathrm{MRI}$ as sessment of iron overload in thalassemia. Int J Med Inform 2009; 78: 503-12.

17. d'Assignies G, Paisant A, Bardou-Jacquet E, et al. Noninvasive measurement of liver iron concentration using 3-Tesla magnetic resonance imaging: validation against biopsy. Eur Radiol 2018; 28: 2022-30.

18. Paisant A, Boulic A, Bardou-Jacquet E, et al. Assessment of liver iron overload by 3 T MRI. Abdom Radiol (NY) 2017; 42: 1713-20.

19. Lainé F, Ruivard M, Loustaud-Ratti V, et al. Metabolic and hepatic effects of bloodletting in dysmetabolic iron overload syndrome: a randomized controlled study in 274 patients. Hepatology 2017; 65: 465-74.

20. Ricchi P, Meloni A, Spasiano A, et al. The impact of liver steatosis on the ability of serum ferritin levels to be predictive of liver iron concentration in non-transfusion-dependent thalassaemia patients. Br J Haematol 2018; 180: 721-6.

21. Rostoker G, Loridon C, Griuncelli M, et al. Liver iron load influences hepatic fat fraction in end-stage renal disease patients on dialysis: a proof of concept study. EBioMedicine 2019; 39: 461-71.

22. Ugarte A, Sánchez-GonzálezJ, Álvarez-de-Eulate C, Alustiza JM, Emparanza JI. Hepatic iron overload estimation by proton density mDIXON Quant technique. Proceedings ISMRM 2018, Abstract 2606.

23. St Pierre TG, El-Beshlawy A, Elalfy M, et al. Multicenter validation of spin-density projection-assisted R2-MRI for the noninvasive measurement of liver iron concentration. Magn Reson Med 2014; 71: 2215-23.

24. Alustiza JM, Emparanza JI, Castiella A, et al. Measurement of liver iron concentration by MRI is reproducible. Biomed Res Int 2015; 2015: 294024.

25. Castiella A, Alustiza JM, Emparanza JI, Zapata EM, Costero B, Diez MI. Liver iron concentration quantification by MRI: Are recommended protocols accurate enough for clinical practice? Eur Radiol 2011; 21: 137-41.

26. Juchems MS, Cario H, Schmid M, Wunderlich AP. Liver iron content determined by MRI: spin-echo vs. gradientecho. Rofo 2012; 184: 427-31.

27. Wunderlich AP, Cario H, Juchems MS, Beer M, Schmidt SA. Noninvasive MRI-based liver iron quantification: methodic approaches, practical applicability and significance. Rofo 2016; 188: 1031-6.

28. Lorcerie B, Audia S, Samson M, et al. Diagnosis of an increased serum level of ferritin. Rev Med Interne 2015; 36: 522-9.

29. Lorcerie B, Audia S, Samson M, et al. Diagnosis of hyperferritinemia in routine clinical practice. Presse Med 2017; 46: e329-38.

30. Roblin X, Chevassus P, Boudemaghe T, Palayodan A. Should the insulin resistance associated with hepatic iron overload be researched during diabetes mellitus type II? Diabetes Metab 2002; 28: 335-9.

31. Adams PC. The (II)logic of iron reduction therapy for steatohepatitis. Hepatology 2015; 62: 668-70.

32. Cullis JO, Fitzsimons EJ, Griffiths WJ, Tsochatzis E, Thomas DW; British Society for Haematology. Investigation and management of a raised serum ferritin. $\mathrm{Br} J$ Haematol 2018; 181: 331-40.

33. Adams LA, Crawford DH, Stuart K, et al. The impact of phlebotomy in non-alcoholic fatty liver disease: a prospective, randomized, controlled trial. Hepatology 2015; 61: 1555-64.

34. Murali AR, Gupta A, Brown K. Systematic review and meta-analysis to determine the impact of iron depletion in dysmetabolic iron overload syndrome and non-alcoholic fatty liver disease. Hepatol Res 2018; 48: e30-41.

35. Adams PC, Barton JC. A diagnostic approach to hyperferritinemia with a non-elevated transferrin saturation. J Hepatol 2011; 55: 453-8.

36. Deugnier Y, Bardou-Jacquet É, Lainé F. Dysmetabolic iron overload syndrome (DIOS). Presse Med 2017; 46: e306-11.

37. Rostoker G, Griuncelli M, Loridon C, et al. Hemodialysis-associated hemosiderosis in the era of erythropoiesis-stimulating agents: an MRI study. Am J Med 2012; 125: 991-9.

38. Castiella A, Zapata E, Alustiza JM. Non-invasive methods for liver fibrosis prediction in hemochromatosis: one step beyond. World J Hepatol 2010; 2: 251-5.

39. Ramm GA, Crawford DH, Powell LW, Walker NI, Fletcher LM, Halliday JW. Hepatic stellate cell activation in genetic haemochromatosis: lobular distribution, effect of increasing hepatic iron and response to phlebotomy. J Hepatol 1997; 26: 584-92.

40. Frazer DM, Anderson GJ, Ramm GA, Subramaniam VN, Powell LW. How much iron is too much? Expert Rev Gastroenterol Hepatol 2008; 2: 287-90.

41. Porto G, Brissot P, Swinkels DW, et al. EMQN best practice guidelines for the molecular genetic diagnosis of hereditary hemochromatosis $(\mathrm{HH})$. Eur J Hum Genet 2016; 24: 479-95. 
42. Pietrangelo A. Non-invasive assessment of hepatic iron overload: are we finally there? J Hepatol 2005; 42: 153-4.

43. Olynyk JK, St Pierre TG, Britton RS, Brunt EM, Bacon BR. Duration of hepatic iron exposure increases the risk of significant fibrosis in hereditary hemochromatosis: a new role for magnetic resonance imaging. Am J Gastroenterol 2005; 100: 837-41.

44. Bassett ML, Halliday JW, Powell LW. Value of hepatic iron measurements in early hemochromatosis and determination of the critical level associated with fibrosis. Hepatology 1986; 6: 24-9.

45. Sallie RW, Reed WD, Shilkin KB. Confirmation of the efficacy of hepatic tissue iron index in differentiating genetic haemochromatosis from alcoholic liver disease complicated by alcoholic haemosiderosis. Gut 1991; 32 207-10.

46. Castiella A, Emparanza J. External validation for fibrosis predicting index in hereditary hemochromatosis. Am J Gastroenterol 2005; 100: 2366-7. 